Meta

Journal des traducteurs

Translators' Journal

\title{
Méthode d'accès informatisé aux combinaisons lexicales en langue technique
}

\section{Marie-Claude L'Homme}

Volume 42, numéro 1, mars 1997

Lexicologie et terminologie

URI : https://id.erudit.org/iderudit/003508ar

DOI : https://doi.org/10.7202/003508ar

Aller au sommaire du numéro

Éditeur(s)

Les Presses de l'Université de Montréal

ISSN

0026-0452 (imprimé)

1492-1421 (numérique)

Découvrir la revue

Citer cet article

L'Homme, M.-C. (1997). Méthode d'accès informatisé aux combinaisons lexicales en langue technique. Meta, 42(1), 15-23.

https://doi.org/10.7202/003508ar
Résumé de l'article

On propose une méthode de codage informatisé des combinaisons lexicales comportant un verbe et un nom. La description des noms se fait sur le modèle des fiches contenues dans les banques de terminologie (fiches monoréférentielles). Quant aux verbes, qui sont pour la plupart polysémiques, leurs distinctions sémantiques sont illustrées à l'aide d'un contexte nominal. L'accès aux combinaisons est réalisé à l'aide d'une interface entre les enregistrements décrivant les deux parties du discours. Le système puise, dans les fiches monoréférentielles, les éléments nominaux et établit un lien avec le contexte nominal qui illustre les changements sémantiques d'une forme verbale. Les liens entre les bases de données sont établis à l'aide d'une structure conceptuelle. 


\title{
MÉTHODE D’ACCÈS INFORMATISÉ AUX COMBINAISONS LEXICALES EN LANGUE TECHNIQUE*
}

\author{
MARIE-Claude L'HOMME \\ Université de Montréal, Canada
}

\begin{abstract}
Résumé
On propose une méthode de codage informatisé des combinaisons lexicales comportant un verbe et un nom. La description des noms se fait sur le modèle des fiches contenues dans les banques de terminologie (fiches monoréférentielles). Quant aux verbes, qui sont pour la plupart polysémiques, leurs distinctions sémantiques sont illustrées à l'aide d'un contexte nominal. L'accès aux combinaisons est réalisé à l'aide d'une interface entre les enregistrements décrivant les deux parties du discours. Le système puise, dans les fiches monoréférentielles, les éléments nominaux et établit un lien avec le contexte nominal qui illustre les changements sémantiques d'une forme verbale. Les liens entre les bases de données sont établis à l'aide d'une structure conceptuelle.
\end{abstract}

\begin{abstract}
This paper proposes a computerized method for encoding verb + noun combinations. Nouns are described according to the model used in terminological databases (monoferential records). As for verbs, which are mostly polysemous, their semantic differences are exemplified in nominal contexts. Verbal and nominal records are linked using the entry in the nominal record, and the nominal context in the verbal record links between records are made using a conceptual categorization of nouns.
\end{abstract}

\section{INTRODUCTION}

L'intérêt manifesté à l'égard des combinaisons lexicales spécialisées (cooccurrents, phraséologismes, collocations ${ }^{1}$ ) n'a fait que croître au cours des quinze dernières années chez les terminologues. Il est né à la suite d'une constatation toute simple: le besoin des traducteurs et des rédacteurs spécialisés de connaître, en vue de les reproduire, des usages particuliers à des langues comme celles de l'économie, des mathématiques ou de l'informatique, pour ne citer que celles-ci.

L'intérêt d'une représentation terminographique des combinaisons lexicales spécialisées ne semble plus faire de doute. Cependant, ces groupes posent encore des problèmes quant au mode de codification à adopter dans les ouvrages de référence, qu'il s'agisse d'ouvrages traditionnels ou d'outils informatisés. Les problèmes se traduisent notamment par l'utilisation d'expressions diverses qui sont rarement définies (collocation, cooccurrent ou phraséologisme) et par l'absence de consensus sur les méthodes de recensement et de codification des combinaisons. De plus, les combinaisons lexicales viennent secouer des modèles terminologiques fondés sur le concept, généralement articulés autour des unités lexicales à vocation nominale. Ces modèles rendent difficile la description d'éléments linguistiques qui se définissent non pas par rapport à un concept, mais par rapport à un usage.

Nous nous attarderons, dans les pages qui suivent, sur un modèle de représentation des combinaisons lexicales ${ }^{2}$. Le modèle proposé veut permettre une consultation informatisée de renseignements liés à des combinaisons de mots utilisées en langue technique. 
Les combinaisons sont retenues dans une perspective de traduction: l'outil est élaboré dans le but de permettre l'accès à de l'information terminographique sur les formes appartenant à deux langues (anglais-français, dans le cas qui nous préoccupe). Le modèle informatisé propose en outre l'intégration d'un outil de référence déjà utilisé par les traducteurs spécialisés, à savoir les banques de données terminologiques.

\section{DONNÉES DE BASE}

\subsection{COMBINAISONS LEXICALES RETENUES}

La méthode d'accès décrite dans les pages qui suivent porte sur des combinaisons d'unités lexicales présentant les caractéristiques suivantes:

1. combinaisons de mots utilisées en langue spécialisée (plus précisément, en langue technique);

2. les unités de la combinaison sont liées grammaticalement;

3. l'emploi des unités lexicales dans un contexte voisin est dicté par des conventions, c'est-à-dire un usage convenu par un groupe de locuteurs (dans le cas qui nous préoccupe, il s'agit d'un groupe de spécialistes);

4. les groupes comprennent: a) une unité terminologique; et b) une autre unité lexicale (un verbe, un adjectif ou un nom) ${ }^{3}$. La deuxième unité lexicale est généralement polysémique;

5. les deux unités lexicales contenues dans une combinaison ont d'autres emplois en langue technique. Les deux unités demeurent autonomes (elles peuvent, entre autres, former d'autres combinaisons créant ainsi tout un réseau combinatoire);

5.1 un verbe peut être employé avec plusieurs unités terminologiques. Par exemple, release peut se combiner à des unités terminologiques diverses:

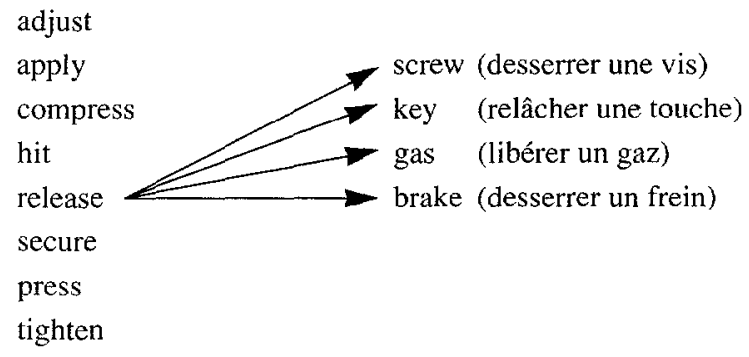

5.2 une unité terminologique peut être utilisée avec plusieurs verbes. Par exemple, screw et key se combinent à des verbes divers:

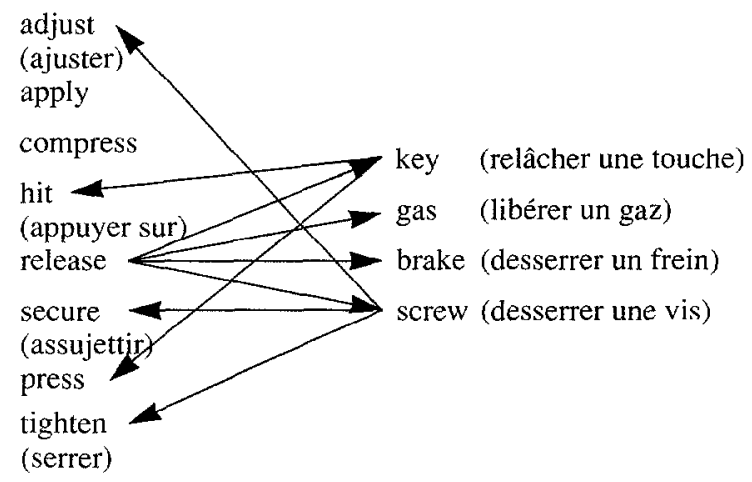


5.3 un verbe ayant un sens donné peut être utilisé avec toute une classe d'unités terminologiques. Par exemple, release, lorsqu'il signifie «desserrer», peut admettre toute une série de termes appartenant à la même classe conceptuelle :

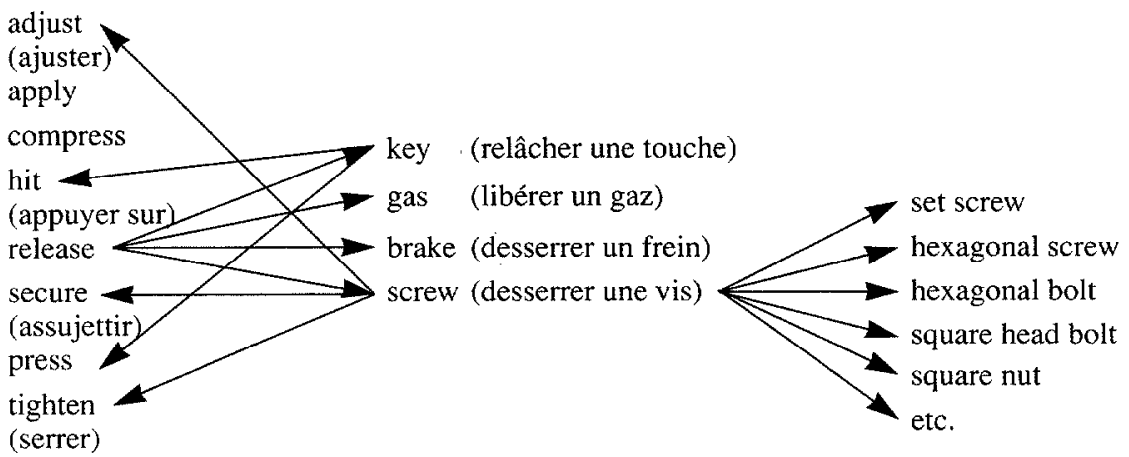

\subsection{UTILITÉ DES COMBINAISONS LEXICALES EN TRADUCTION SPÉCIALISÉE}

En pratique, le recensement et la représentation des combinaisons lexicales spécialisées (dans les ouvrages de référence informatisés ou non) permet au traducteur de connaître un usage donné dans une langue de spécialité en vue de le reproduire dans une langue d'arrivée.

Le traducteur peut, en outre, chercher les équivalents et les explications rattachés à un verbe polysémique (release (gas): libérer un gaz; release (brake): desserrer les freins, etc.). Les recherches d'un traducteur peuvent également porter sur les équivalents d'une forme verbale dans les cas où les langues pratiquent des découpages différents de la réalité (ainsi, fail se traduit différemment en français en fonction de l'objet qui «fait défaut»). Enfin, un traducteur peut vouloir connaître l'ensemble des verbes qui sont utilisés avec une unité terminologique donnée afin de faire des choix éclairés (p. ex. Quels sont les verbes utilisés avec frein? : serrer, desserrer, etc.).

\subsection{OBJECTIFS TECHNIQUES DU MODÈLE D'ACCÈS AUX \\ COMBINAISONS LEXICALES SPÉCIALISÉES}

Un programme informatique doit évidemment permettre l'interrogation et l'accès à des combinaisons lexicales spécialisées répondant aux critères énumérés plus haut. L'accès devrait être permis: 1) à partir du verbe (p. ex. Quels sont les termes utilisés avec ce verbe ?) ; 2) à partir du terme (p. ex. Quels sont les verbes utilisés avec ce terme ?); ou 3) à partir des deux éléments de la combinaison (p. ex. Quelle est la traduction d'une combinaison verbe + terme donné?; ou: Que signifie ce verbe lorsqu'il est utilisé avec un terme donné ?).

Des accès comme ceux qui sont proposés ci-dessus sont facilement réalisables au moyen d'un outil informatique. Le projet élaboré comporte toutefois d'autres objectifs.

Un de ces objectifs additionnels consiste à permettre l'ajout d'information lexicographique pour les deux éléments de la combinaison. Cette information lexicographique peut être composée d'une définition, d'exemples, de synonymes, de variantes ou de notes. En ce qui concerne les termes, des renseignements du genre sont déjà fournis sur les fiches de terminologie traditionnelles (voir ci-dessous). Pour ce qui touche les formes verbales, le modèle informatique devrait permettre l'ajout de renseignements qui rendent compte de leurs acceptions techniques.

Le dernier objectif visé consiste à intégrer un outil de travail déjà utilisé par les traducteurs spécialisés, à savoir les banques de terminologie (ou bases de données 
terminologiques). Cette méthode permet de récupérer des données compilées sur un des éléments de la combinaison lexicale, à savoir le terme ${ }^{4}$. Le programme doit donc permettre l'accès aux combinaisons lexicales en passant par la fiche terminologique standard et, à l'inverse, permettre la consultation d'une fiche en passant par une combinaison lexicale. Nous voulions également offrir à un utilisateur éventuel la possibilité de ne consulter que la composante qui l'intéresse au cours d'une séance d'interrogation, sans avoir à se préoccuper de la seconde s'il ne le souhaite pas.

\section{SCÉNARIO RETENU}

Nous avons envisagé différentes méthodes de codification des combinaisons lexicales. En fait, plusieurs solutions sont possibles (les combinaisons lexicales en entrée, ajout de champs dans la fiche de terminologie standard, etc.). Nous avons retenu le modèle décrit plus bas, car il permet d'atteindre les objectifs cités précédemment et de gérer de façon intelligente et économique des combinaisons lexicales techniques. Les avantages qu'il présente sont décrits en conclusion.

Notre modèle consiste donc, dans une première étape, à créer une base de données parallèle décrivant les acceptions techniques des unités verbales et, en second lieu, à établir un lien entre cette base verbale et une base de données terminologiques classique. Nous décrirons d'abord la base verbale; l'explication du lien entre la base verbale et la base terminologique suivra.

La base verbale prend la forme suivante. Chaque verbe est décrit en fonction d'une grille prédéfinie tenant compte, dans une première partie, des éléments suivants :

1) structures syntaxiques dans lesquelles les verbes peuvent se retrouver en langue technique;

2) une ou plusieurs définitions;

3) exemples;

4) renvois (synonymiques, antonymiques).

Cette liste de renseignements n'est pas limitative: elle correspond plutôt à notre perception de ce qui devrait figurer dans des fiches verbales pour rendre compte de leurs acceptions techniques.

Des renseignements identiques peuvent être fournis pour les formes verbales des deux langues. Toutes les acceptions d'un verbe apparaissent dans un enregistrement distinct. Une acception nouvelle est admise en fonction de deux critères $\left.{ }^{5}: 1\right)$ une forme verbale a des sens différents qui mènent ou non à des équivalents différents;2) il existe des cas où les langues pratiquent des découpages différents de la réalité, entraînant des choix d'équivalents différents.

La grille utilisée est largement inspirée des articles présentés dans un dictionnaire monolingue, le Collins Cobuild ${ }^{6}$. L'exemple figurant ci-dessous rend compte d'un certain nombre d'acceptions du verbe run dans le domaine de l'informatique.

1. RUN V + O Start something and continue it until he results. The operating system runs applications. SYN. execute

SUJET : person; command; computer

OBJET : $\quad$ command

F. exécuter $\quad \mathrm{V}+\mathrm{O}$

SUJET : person : command ; computer

OBJET : command 
2. RUN V + A(on) One version, called A / UX, runs on powerful Macintosh systems.

SUJET : command

CIRCONSTANT : computer

F. tourner $\quad \mathrm{V}+\mathrm{A}(\mathrm{sur})$ Windows 3 pouvait tourner sur des ordinateurs équivalents.

SUJET : command

CIRCONSTANT : computer

3. RUN V To make something work. ((...)) and assist in helping the computer run more effectively $((\ldots))$

$$
\begin{array}{lcl} 
& \text { SUJET }: \text { computer } \\
\text { F. tourner } & \mathrm{V} & \\
& \text { SUJET }: \text { computer }
\end{array}
$$

4. RUN V + O The POST runs a series of tests $((\ldots))$

SUJET : person; computer; command

OBJET : activity

F. effectuer

$\mathrm{V}+\mathrm{O}$

SUJET : person; computer; command

OBJET : activity

En plus des renseignements cités ci-dessus, les enregistrements comportent une seconde partie. Celle-ci renferme un certain nombre de champs rendant compte des termes avec lesquels un verbe peut être combiné. Nous avons recours à un système d'étiquettes qui figurent dans les champs correspondant à la fonction des noms (Sujet, Objet ou Circonstant $)^{7}$.

Un traducteur qui consulte une fiche verbale construite de cette façon sait, en voyant l'étiquette apparaissant dans les champs appropriés, si le sens du verbe et son contexte d'application correspondent au cas qu'il doit régler.

C'est précisément l'étiquette nominale qui servira à établir un lien entre les fiches verbales et une base de données terminologiques. Comme l'étiquette figurant dans les champs (SUJET/OBJET/CIRCONSTANT) renvoie à la classe conceptuelle des termes admis dans l'environnement d'un verbe, elle permet de regrouper des ensembles de termes en définissant la classe d'objets à laquelle ils appartiennent. Par exemple, la deuxième acception de run citée plus haut comporte les étiquettes command et computer apparaissant respectivement dans les champs sujet et circonstant. L'exemple est reproduit ci-dessous :

2. RUN V + A(on) One version, called A/UX, runs on powerful Macintosh systems.

SUJET : command

CIRCONSTANT : computer

F. tourner $\quad \mathrm{V}+\mathrm{A}(\mathrm{sur})$ Windows 3 pouvait tourner sur des ordinateurs équivalents.

SUJET : command

CIRCONSTANT : computer

La fiche permet de conclure que la forme verbale run, dans une de ses acceptions, peut admettre comme sujet des unités terminologiques appartenant à la classe conceptuelle des «command». Il s'agit d'une étiquette utilisée pour regrouper toutes les composantes logicielles (système d'exploitation, logiciel d'application, etc.). Le verbe admet également des circonstants qui appartiennent à la classe des «computer». Cette étiquette regroupe toutes les unités terminologiques désignant des types d'ordinateurs (miniordinateur, micro-ordinateur, ordinateur portable, etc. $)^{8}$. 

de run:

Les unités terminologiques suivantes peuvent donc apparaître dans l'environnement

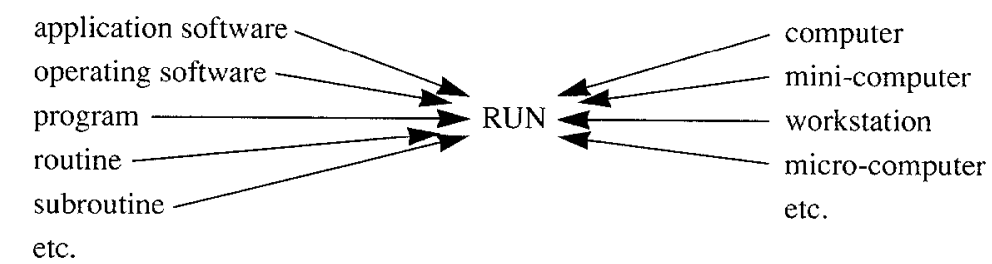

Les entrées de la base terminologique sont étiquetées au moyen du même système. Ainsi, tous les termes appartenant à la classe des command (application software, operating software, program, routine, etc.) sont marqués. Ce qui signifie que si, d'une part, une acception de run admet des sujets appartenant à la classe des command et que, d'autre part, application software est défini comme un command, les éléments dans les deux bases sont liés. Application software peut donc être utilisé comme sujet de run dans une de ses acceptions.

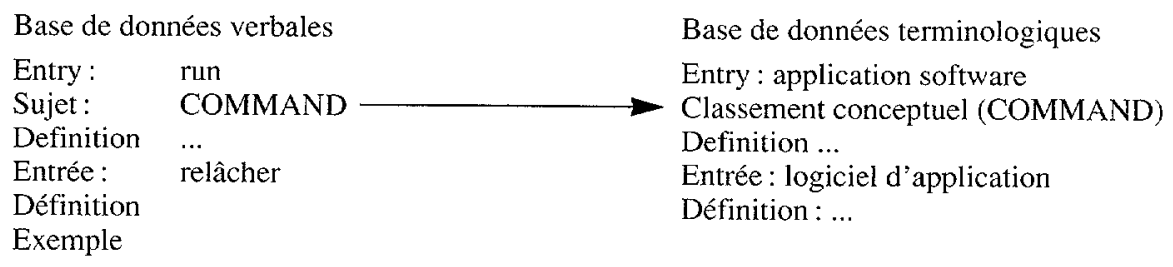

Les entrées terminologiques sont toutes marquées au moyen d'un système d'étiquettes repris dans la base verbale. De cette manière, elles peuvent être associées à des séries de verbes comportant des étiquettes identiques. Par exemple, un terme comme microcomputer, qui appartient à la classe des computer, peut être combiné avec run dans les quatre acceptions citées plus haut: dans trois cas comme sujet, dans un cas comme circonstant.

$$
\begin{aligned}
& \text { (command) runs on a micro-computer } \\
& \text { the micro-computer runs (command) } \\
& \text { the micro-computer runs effectively } \\
& \text { the micro-computers runs a (activity) }
\end{aligned}
$$

Notre modèle permet de lier des unités lexicales et de renseigner un utilisateur éventuel sur les possibilités combinatoires de ces unités. Il permet également la consultation de rubriques complémentaires sur les formes verbales et leurs acceptions en langue technique. Il permet, enfin, de parcourir les fiches terminologiques que l'utilisateur a l'habitude de rechercher ou des fiches verbales de façon tout à fait indépendante.

\section{CONCLUSION}

Le modèle décrit dans les pages qui précèdent a déjà été mis à l'essai sur un corpus d'entrées terminologiques et une centaine de verbes liés à la langue de l'informatique. Les bases doivent être enrichies ${ }^{9}$, mais il est déjà possible d'entrevoir les possibilités du modèle.

Bien entendu, les liens établis entre les deux bases de données entraînent un marquage systématique des entrées dans les bases terminologiques classiques. Cependant, le modèle n'affecte pas en profondeur la forme actuelle des fiches de terminologie. Par ailleurs, il 
est de plus en plus question, dans les travaux récents de terminologie, de rendre compte des liens conceptuels entre les entrées. Un marquage semblable à celui dont il est question ici sera pratiqué à d'autres fins, mais pourra être réutilisé ici.

Le principal avantage du modèle présenté réside dans le fait qu'il n'implique pas une identification préalable des combinaisons lexicales dites «significatives», c'est-à-dire susceptibles de faire l'objet de recherches par l'utilisateur. Cette identification poserait toutes sortes de problèmes au stade d'entrée des données. En outre, le choix des combinaisons lexicales pose des problèmes théoriques qui n'ont pas encore été tranchés par les terminologues.

En outre, l'utilisation de bases différentes pour rendre compte des acceptions terminologiques et des acceptions verbales évite les répétitions. Dans ce modèle, chaque forme a un nombre de descriptions qui correspond à ses acceptions en langue technique.

L'utilisation des étiquettes représentant des classes conceptuelles permet d'éviter de dresser un inventaire des termes pouvant apparaître dans l'environnement d'un verbe. Par exemple, release, dans une de ses acceptions techniques, admet des termes appartenant à la classe button (classe comprenant les boutons et les touches). Il se traduit alors par relâcher ${ }^{10}$. Si les liens étaient établis entre des formes linguistiques, il faudrait énumérer toutes les formes susceptibles d'apparaître dans l'environnement de release, lorsqu'il a le sens de «relâcher». En revanche, l'étiquette utilisée dans notre modèle regroupe toutes les formes pouvant être combinées à la forme verbale.

$\begin{array}{ll}\text { release } & \text { enter key } \\ \text { control key } & \text { key } \\ \text { computer key } & \text { pushbutton } \\ \text { button } \\ \text { etc. }\end{array}$

release (button)

Enfin, le modèle facilite grandement les mises à jour ultérieures qui peuvent être faites dans chacune des bases de données (verbale et terminologique). Par exemple, si un terme est ajouté à la base de données terminologiques, il suffit de lui attribuer une étiquette correspondant à sa catégorisation conceptuelle, et tous les verbes qui comportent une étiquette correspondante lui sont immédiatement associés. Par exemple, si un nouveau type de touche fait son apparition sur les claviers, il suffira de l'entrer dans une base terminologique, de la décrire et de lui attribuer l'étiquette button. Cette nouvelle entrée sera immédiatement associée à des verbes comme release, press, depress, hit, etc., qui comportent l'étiquette button.

\section{Notes}

* Cet article est issu d'une communication présentée par l'auteur aux IVes Journées scientifiques du réseau «Lexicologie, terminologie, traduction» de l'AUPELF-UREF (Lyon, France, 28, 29, 30 septembre 1995).

1. Les expressions collocation, cooccurrent ou phraséologisme, largement véhiculées ces dernières années dans les travaux terminologiques, ne seront pas utilisées ici. Les sens prêtés à ces différentes expressions varient sensiblement en terminologie. En outre, les groupes retenus diffèrent des ensembles généralement décrits par les lexicographes qui ont créé les expressions. Nous aurons recours aux désignations combinaison lexicale ou combinaison de mots qui sont, nous l'espérons, plus neutres.

2. Cette recherche est réalisée à l'Université de Montréal grâce à la collaboration d'étudiants de $2^{\mathrm{e}}$ cycle : René Gemme et Isabelle Laporte. L'équipe composée des étudiants cités et de l'auteur est en train de mettre au point le système informatique intégrant la méthode dont il est question dans ces pages. Les travaux sont menés dans le cadre d'une subvention accordée par le Fonds FCAR. 
3. Dans une première étape, nous avons retenu les groupes composés d'une unité terminologique et d'un verbe (le terme pouvant assumer différentes fonctions: $\mathrm{V}+\mathrm{N}, \mathrm{N}+\mathrm{V}, \mathrm{V}+$ prep $+\mathrm{N}$ ). Cependant, le modèle peut s'appliquer à d'autres combinaisons lexicales dans lesquelles figure un terme.

4. Rappelons brièvement quelques principes généraux des bases de données terminologiques. Ces outils de référence utilisent une approche dite «uniconceptuelle». En pratique, cela signifie qu un enregistrement figurant dans la base rend compte d'un seul concept. Compte tenu de cette approche uniconceptuelle, la banque de terminologie est particulièrement bien adaptée à la description de certains types de noms (l. les noms monosémiques, quel que soit le domaine de spécialité; 2 . les noms polysémiques, mais dont les acceptions peuvent être associées à des domaines distincts). Les noms constituent de toute manière la plus grande proportion des unités décrites dans les banques. Les bases de données terminologiques sont généralement bilingues ou multilingues. Cela signifie, par conséquent, que les formes appartenant à différentes langues représentées sur une fiche renvoient nécessairement à un concept unique.

5. Il est possible qu'un troisième critère devienne essentiel. Bien qu'il ne nous ait pas encore été permis de l'observer, une distinction par domaine de spécialité pourrait se révéler nécessaire.

6. Pour une explication plus détaillée, voir L'Homme (1995b).

7. On peut se demander pourquoi les étiquettes figurent dans les deux parties de l'enregistrement (anglaise et française), puisque les codes sont les mêmes. Nous les reprenons pour tenir compte des cas où les structures syntaxiques diffèrent d'une langue à l'autre (p. ex. press $(\mathrm{V}+\mathrm{O})$ : press a key; appuyer $(\mathrm{V}+\mathrm{A}(\mathrm{sur})$ : appuyer sur une touche $)$.

8. Les classes conceptuelles et leurs étiquettes sont inspirées d'un modèle mis au point par Sager et Kageura (1994-1995) que nous élaborons constamment. Le modèle de Sager et Kageura est semi-hiérarchisé et comprend quatre grandes catégories (entity concepts, activity concepts, relation concepts et properfy concepts) auxquelles sont subordonnées des catégories moins grandes (entity concepts : material entities, abstract entities, representational entities, etc.).

9. Nous comptons, entre autres, étendre le corpus à d'autres domaines techniques et tenir compte d'autres combinaisons lexicales dans lesquelles figurent un terme $(\mathrm{N}+$ préposition + terme et terme + adjectif).

10. L'exemple est cité au début de l'article.

\section{RÉFÉRENCES}

ATTALI, A., BOURQUIN, G. et al. (1992) : «Aide au transfert lexical dans une perspective de TAO : expérimentation sur un lexique non terminologique, Meta, 37 (4), pp. $770-790$.

BÉJOINT, H. et Ph. THOIRON (1992): «Macrostructure et microstructure dans un dictionnaire de collocations en langue de spécialité», Terminologie et traduction, 2/3, pp. 513-522.

BENSON, M., BENSON, E. and R. ILSON (1986): The BBI Combinatory Dictionary of English: A Guide to World Combinations. Amsterdam. Philadelphia, John Benjamins.

BLAMPAIN, D. (1993) : «Notions et phraséologie. Une nouvelle alliance ?», Phraséologie. Actes du Séminaire international, Terminologies notvelles, 10, pp. 43-49.

CLAS, A. (1994): «Collocation et langues de spécialité», Mefa, 39 (4), pp. 576-580.

COHEN, B. (1986) : Lexique de cooccurrents. Bourse - Conjoncture économique, Montréal, Linguatech.

COHEN, B. (1992) : «Méthodes de repérage et de classement des cooccurrents lexicaux», Terminologie er traduction, 2/3, pp. 505-511.

Collin Cobuild. English Language Dictionary (1987) : London, Glasgow, Collins.

FELLBAUM, C. (1994): «English Verbs as a Semantic Net», International Jou'nal of Lexicography, 3 (4), pp. 278-301.

GROSS, G. (1994) : «Classes d'objets et description des verbes», Languages, 115, pp. 15-30.

HEID, U. (1992): «Décrire les collocations. Deux approches lexicographiques et leur application dans un outil informatisé», Terminologie et traduction, 2/3, pp. 523-548.

HEID. U. et G. FREIBOTT (1991) : «Collocations dans une base de données terminologique et lexicale», Meta, $36(1)$, pp. 77-91.

L'HOMME, M.-C. (1992) : «Les unités phraséologiques et leurs représentations en terminographie», Terminologie et traduction, $2 / 3$, pp. $493-503$.

L'HOMME, M.-C. (1995a): «Processing Word Combinations in Existing Term Banks», Terminology, 2 (1), pp. $141-162$

L'HOMME, M.-C. (1995b) : «Définition d'une méthode de recensement et de codage des verbes en langue technique : applications en traduction», à paraître dans Tiaduction, terminologie et rédaction (TTR).

PAVEL, S. (1993): «Vers une méthode de recherche phraséologique en langue de spécialité», L'Actualité terminologique, 26 (2), pp. 9-13.

PICHT, H. (1987) : «Terms and their LSP Environment - LSP Phraseology», Meta, 23 (2), pp. 149-155.

ROBERTS, R. (1993) : «Phraseology: The State of the Art», Terminology Update, 26 (2), pp. 4-8. 
ROBERTS, R. (1994-1995) : «Identifying Phraseology of Language for Special Purposes», Terminology and LSP Linguistics. Studies in Specialized Vocabularies and Texts. Actes de Langue française et de linguistique (ALFA), 7/8, pp. 61-74.

SAGER, J. C. (1990) : A Practical Course in Terminology Processing, Amsterdam, Philadelphia, John Benjamins.

SAGER, J. C. and K. KAGEURA (1994-1995) : «Concept Classes and Conceptual Structure - Their Role and Necessity in Terminology», Terminology and LSP Linguistics. Studies in Specialized Vocabularies and Texts. Actes de Langue française et de linguistique (ALFA), 7/8, pp. 191-216.

SAGER, J. C. et M.-C. L'HOMME (1994) : «A Model for the Definition of Concepts: Rules for Analytical Definitions in Terminological Databases», Terminology, 1 (2), pp. 373-395. 